\title{
Resveratrol enhances cisplatin-induced apoptosis in human hepatoma cells via glutamine metabolism inhibition
}

\author{
Zhaoyuan Liu ${ }^{1}$, Qing Peng ${ }^{1}$, Yang $\mathrm{Li}^{1} \mathcal{E}$ Yi Gao ${ }^{1,2, *}$ \\ ${ }^{1}$ Department of Hepatobiliary Surgery II, Guangdong Provincial Research Center for Artificial Organ and Tissue Engineering, Guangzhou \\ Clinical Research and Transformation Center for Artificial Liver, Institute of Regenerative Medicine, Zhujiang Hospital, Southern Medical \\ University, Guangzhou 510280, Guangdong Province, ${ }^{2}$ State Key Laboratory of Organ Failure Research, Southern Medical University, \\ Guangzhou 510515, China
}

Cisplatin is one of the most effective chemotherapeutic drugs used in the treatment of HCC, but many patients will ultimately relapse with cisplatin-resistant disease. Used in combination with cisplatin, resveratrol has synergistic effect of increasing chemosensitivity of cisplatin in various cancer cells. However, the mechanisms of resveratrol enhancing cisplatininduced toxicity have not been well characterized. Our study showed that resveratrol enhances cisplatin toxicity in human hepatoma cells via an apoptosis-dependent mechanism. Further studies reveal that resveratrol decreases the absorption of glutamine and glutathione content by reducing the expression of glutamine transporter ASCT2. Flow cytometric analyses demonstrate that resveratrol and cisplatin combined treatment leads to a significant increase in ROS production compared to resveratrol or cisplatin treated hepatoma cells alone. Phosphorylated $\mathrm{H} 2 \mathrm{AX}(\gamma \mathrm{H} 2 \mathrm{AX})$ foci assay demonstrate that both resveratrol and cisplatin treatment result in a significant increase of $\gamma \mathrm{H} 2 \mathrm{AX}$ foci in hepatoma cells, and the resveratrol and cisplatin combined treatment results in much more $\gamma \mathrm{H} 2 \mathrm{AX}$ foci formation than either resveratrol or cisplatin treatment alone. Furthermore, our studies show that over-expression of ASCT2 can attenuate cisplatin-induced ROS production, $\gamma \mathrm{H} 2 \mathrm{AX}$ foci formation and apoptosis in human hepatoma cells. Collectively, our studies suggest resveratrol may sensitize human hepatoma cells to cisplatin chemotherapy via glutamine metabolism inhibition. [BMB Reports 2018; 51(9): 474-479]

${ }^{*}$ Corresponding author. Tel: +86-0206143207; Fax: +86-02061432; E-mail: drgaoy@126.com

https://doi.org/10.5483/BMBRep.2018.51.9.114

Received 23 May 2018, Revised 23 June 2018, Accepted 8 August 2018

Keywords: Apoptosis, Cisplatin, Glutaminolysis, Human hepatoma cells, Resveratrol

\section{INTRODUCTION}

Hepatocellular carcinoma ( $\mathrm{HCC}$ ) is one of the most common malignant tumors in the world. The main treatment for HCC is surgical resection, but only $10 \%$ to $15 \%$ of patients with HCC are candidates for surgical resection (1). Randomized controlled trials have gradually supported and established TACE based comprehensive interventional therapy as the preferred method for non-surgical treatment of HCC (2-4). Cisplatin (CDDP) is one of the most effective chemotherapeutic drugs used in the treatment of HCC, but development of resistance limits the successful use of cisplatin in chemotherapy (5). Therefore, there is a critical need for finding other drugs that can enhance the antitumor activity of CDDP. Resveratrol (RV) is a non-flavonoid polyphenol belonging to the stibenes. RV has been shown to be a potential chemopreventive agent $(6,7)$. Combining with cisplatin, resveratrol had additive and/or synergistic effects increasing the chemosensitisation of cancer cells. However, the mechanisms by which RV increases the chemosensitivity of CDDP remain to be determined.

From the perspective of altered metabolism in cancer cells, apart from glucose, glutamine is another major source of energy production for cancer cells (8) and their metabolism is required in cancer cell growth and survival (9), and resveratrol has been shown to inhibit glucose uptake $(10,11)$. Loss of glutamine influx may inhibit glutathione synthesis resulting in the induction of apoptosis (12). CDDP can induce apoptosis and inhibit cancer cell proliferation through various signaling pathways (13). Therefore, the goal of this study was to determine if resveratrol could sensitize hepatoma cell lines to cisplatin-induced cytotoxicity by inhibiting glutamine metabolism.

\section{RESULTS}

Resveratrol enhances cisplatin toxicity in human hepatoma cell lines via an apoptosis-dependent mechanism

First of all, we evaluated the impact of different doses of RV or CDDP on two hepatoma cells. The results show that cell growth of $\mathrm{C} 3 \mathrm{~A}$ and SMCC7721 were inhibited in a dose-dependent manner by RV and CDDP (Fig. 1A, B). Previous study showed that RV treatment increased the sensitivity of epithelial ovarian cancer 
cells to CDDP induced cell death (15). Here, we sought to investigate whether RV treatment could sensitize human hepatoma cells to CDDP-induced cell toxicity. We chose the lowest two doses of RV and CDDP to treat C3A and SMCC7721 cells for $24 \mathrm{~h}$. The MTS results show that RV enhances CDDP induced cell death in C3A and SMCC7721 cells, and the comparison of cytotoxic effect of $12.5 \mathrm{ug} / \mathrm{ml} \mathrm{RV}$ and $0.625 \mathrm{ug} / \mathrm{ml}$ CDDP vs $0.625 \mathrm{ug} / \mathrm{ml}$ CDDP were more substantial than the other groups, although $\mathrm{CDI}<1$ was measured in all groups (Fig. 1C, D). Moreover, resveratrol did not enhance the inhibitory effect of cisplatin on the growth of normal hepatic cell LO2.

Furthermore, we investigated whether RV could promote CDDP induced apoptosis because CDDP primarily induces cell death by apoptosis, and resveratrol was also identified as a powerful proapoptotic agent. The results showed that $12.5 \mathrm{ug} / \mathrm{ml} \mathrm{RV}$ enhances $0.625 \mathrm{ug} / \mathrm{ml}$ CDDP induced apoptosis in C3A and SMCC7721 cells (Fig. 1F, G).

\section{Resveratrol inhibits glutamine metabolism in human hepatoma cell lines}

RV has been shown to inhibit glucose upake (10). In this study, we examined whether resveratrol reduced glutamine absorption by inhibiting glutamine membrane transporter ASCT2. We tested the effect of resveratrol on the ASCT2 expression of C3A and SMCC7721 cells by western blot. The results showed that resveratrol significantly reduced the ASCT2 expression of C3A and SMCC7721 cells (Fig. 2A). Then we tested glutamine uptake and glutathione content of $\mathrm{C} 3 \mathrm{~A}$ and SMCC7721 cells treated CDDP with or without RV treatment. The results showed that resveratrol



Fig. 1. Resveratrol promotes cisplatin-induced cell death in $C 3 A$ and SMCC7721 cells. (A) MTS assays show that cell growth of C3A and SMCC7721 were inhibited in a dose-dependent manner after treated with resveratrol for $24 \mathrm{~h}$. (B) MTS assays show that cell growth of C3A and SMCC7721 were inhibited in a dose-dependent manner after treated with cisplatin for $24 \mathrm{~h}$. (C) Resveratrol enhances cisplatin induced cell death in C3A cells. (D) Resveratrol enhances cisplatin induced cell death in SMCC7721 cells. (E) Resveratrol does not enhance cisplatin induced cell death in normal hepatic cell LO2. (F) Resveratrol enhances cisplatin induced apoptosis in $\mathrm{C} 3 \mathrm{~A}$ and SMCC7721 cells. CDI $<1$ of All groups. ${ }^{* P}<0.05, * * P<0.01$, $* * * \mathrm{P}<0.0001$. (g) Resveratrol enhances cisplatin induced cell death was quantified by staining with propidium iodide (PI) and Annexin V. significantly reduced glutamine uptake and decreases glutathione content in C3A and SMCC7721 cells (Fig. 2B, C). These results suggest that the mechanism of resveratrol improving the anti-tumor activity of cisplatin may be related to its ability of significantly inhibiting glutamine metabolism of tumor cells.

\section{Resveratrol increases ROS production in human hepatoma cell lines}

We performed flow cytometric analysis to measure ROS production in cisplatin treated C3A and SMCC7721 cells with or without reveratrol treatment for 24 hours. The results showed that resveratrol and cisplatin combined treatment markedly increases ROS production in C3A and SMCC7721 cells compared with those cells treated with resveratrol or cisplatin alone (Fig. 2D, E). These results suggest that increasing of ROS production may be a key role played by resveratrol to enhance cisplatin toxicity.

\section{Resveratrol increases DNA damage in human hepatoma cell lines}

Given that DNA damage is the major cause of cisplatin- induced toxicity, we hypothesized that resveratrol may enhance $\gamma \mathrm{H} 2 \mathrm{AX}$-induced DNA damage To test this hypothesis, we performed $\gamma \mathrm{H} 2 \mathrm{AX}$ foci assay to examine whether RV treatment enhances DNA damage in CDDP treated human hepatoma cells. The results showed that resveratrol and cisplatin combined treatment results in more $\gamma \mathrm{H} 2 \mathrm{AX}$ foci formation than resveratrol and cisplatin treatment alone (Fig. 2F, G).

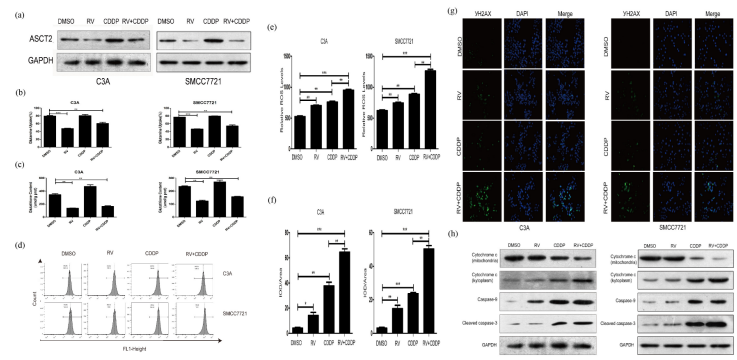

Fig. 2. Resveratrol enhances cisplatin-induced toxicity via a DNA damage causing apoptosis-independent mechanism. (A) Western blot was performed to determine the expression levels of glutamine transporter ASCT2 in C3A and SMCC7721 cells after treated with indicated drugs. $\beta$-Actin was used as a loading control. (B) Resveratrol inhibits the uptake of glutamine in $\mathrm{C} 3 \mathrm{~A}$ and SMCC7721 cells. (C) Resveratrol decreases glutathione content in $\mathrm{C} 3 \mathrm{~A}$ and SMCC7721 cells. (D) Levels of ROS were measured by flow cytometric analyses in $\mathrm{C} 3 \mathrm{~A}$ and SMCC7721 cells. (E) Relative ROS levels in $\mathrm{C} 3 \mathrm{~A}$ and SMCC7721 cells are shown compared to control cells. (F) Quantification of $\gamma \mathrm{H} 2 \mathrm{AX}$ immunofluorescent staining by IOD/Area of C3A and SMCC7721 cells. (G) Picture of $\gamma \mathrm{H} 2 \mathrm{AX}$ immunofluorescent staining of $\mathrm{C} 3 \mathrm{~A}$ and SMCC7721 cells. $(\mathrm{H})$ Western blott assays were performed to determine the expression levels of mitochondria and kytoplasm cytochrome c, caspase-9 and cleaved caspase-3 in $\mathrm{C} 3 \mathrm{~A}$ and SMCC7721 cells. $\beta$-Actin was used as a loading control. $* P<0.05, * * P<0.01, * * * P<0.0001$. 
CDDP-induced DNA damage will ultimately trigger apoptotic pathways (16). In mitochondrial apoptotic pathways, ROS and DNA activate bcl-2 to promote cytochrome c release. To address this issue, western blot analyses were performed to determine the expression level of mitochondria and kytoplasm cytochrome c, caspase- 9 and activated caspase- 3 . The results show that RV treatment has significant effect on the expression of mitochondria and kytoplasm cytochrome c, caspase- 9 and activated caspase-3 (Fig. 2H). These results support the hypothesis that the effect of resveratrol enhancing cisplatin toxicity may be related to increasing ROS-induced DNA damage.

Resveratrol decreases the absorption of glutamine by reducing the expression of ASCT2 and improved the anti-tumor activity of cisplatin

To determine the role of glutamine metabolism in RV-mediated CDDP chemosensitization, we transfect pcDNA3.1-ASCT2 into C3A and SMCC7721 cells. Western blot results indicate that the transfected ASCT2 eukaryotic expression vector increases ASCT2 expression in C3A and SMCC7721 cells compared to normal cell and empty vector (Fig. 3A, B). After transfection of ASCT2 expression vectors in $\mathrm{C} 3 \mathrm{~A}$ and SMCC7721 cells, glutamine metabolism, ROS production, DNA damage and expression of apoptosis-regulating proteins were significantly attenuated (Fig.
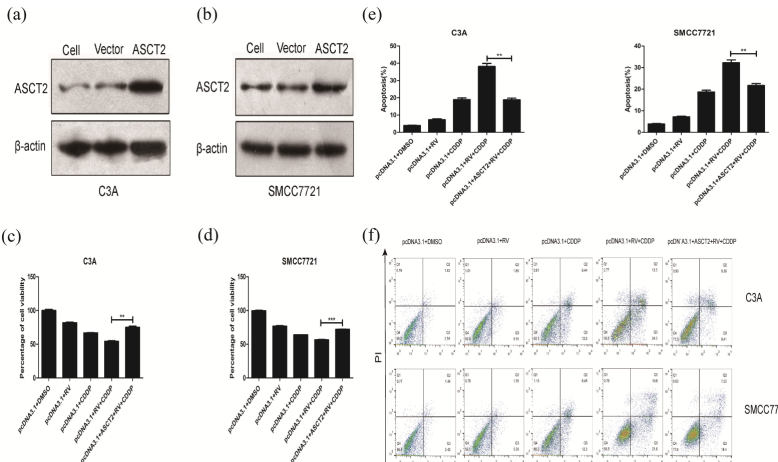

(d)
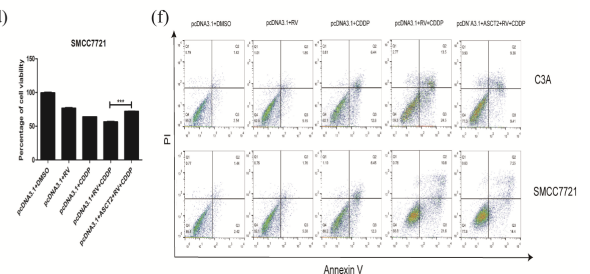

Fig. 3. Enhanced expression of ASCT2 inhibited the synergistic effect of resveratrol on the toxicity of cisplatin on C3A and SMCC7721 cells. (A) C3A cells were transfected with empty vector pcDNA3.1 or pcDNA3.1 + ASCT2. Transfection efficiency is confirmed by Western blot assay. (B) SMCC7721 cells were transfected with empty vector pcDNA3.1 or pcDNA3.1+ASCT2. Transfection efficiency is confirmed by Western blot assay. (C) Transfection of ASCT2 vector inhibited the synergistic effect of resveratrol on cisplatin-induced cell death in C3A cells. (D) Transfection of ASCT2 vector inhibited the synergistic effect of resveratrol on cisplatin-induced cell death in SMCC7721 cells. (E) Transfection of ASCT2 vector inhibited the synergistic effect of resveratrol on cisplatin-induced apoptosis in C3A cells. (E) Transfection of ASCT2 vector inhibited the synergistic effect of resveratrol on cisplatin-induced apoptosis in SMCC7721 cells. (F) Transfection of ASCT2 vector inhibited the synergistic effect of resveratrol on cisplatin-induced cell death, which was quantified by staining with propidium iodide (PI) and Annexin V. ${ }^{* * P}<0.01$, ***P $<0.0001$.
4A-G). Moreover, after the recovery expression of ASCT2, the synergistic effect and apoptosis induced effects of resveratrol were lost to cisplatin (Fig. 3C-F). These results prove that ASCT2 is the molecular target of resveratrol. By down-regulating the expression of ASCT2 resulting in inhibiting glutamine metabolism of human hepatoma cell lines, resveratrol improves the sensitivity of tumor cells to cisplatin.

\section{DISCUSSION}

Cisplatin is a neutral, square-planar, coordination complex of divalent $\mathrm{Pt}$ (17). Cisplatin is widely used in the treatment of solid tumors. The use of cisplatin is limited by its severe side effects due to dose-limiting toxicity especially nephrotoxicity, which involves mechanisms include cell cycle arrest, generation of reactive oxygen species (ROS) and apoptosis (18). Another limitation for the use of cisplatin is the development of resistance during the course of the treatment (5). Combination therapies of cisplatin with other drugs have been highly considered to reduce toxicity. Resveratrol, a phytoalexin found in many plants including grapes, berries and peanuts, is a nutraceutical that has many exciting pharmacological potential. Largeamount of studieshave shown the chemopreventive effects of resveratrol on cancers in vitro and in vivo. Currently, resveratrol has been reported to show synergistic effects with cisplatin as a result of increased apoptosis associated with the release of cytochrome $\mathrm{c}$ from mitochondria to cytosol, and the abnormal expression of $\mathrm{Bcl}-2$ and Bax proteins (19). However, the chemopreventive mechanisms of resveratrol



Fig. 4. Enhanced expression of ASCT2 lowered the inhibition of glutaminemetabolism induced by resveratrol. (A) Transfection of ASCT2 vector lowered the inhibition of glutamine uptake induced by resveratrol. (B) Transfection of ASCT2 vector increased glutathione content in $\mathrm{C} 3 \mathrm{~A}$ and $\mathrm{SMCC} 7721$ cells treated with resveratrol. (C) ROS levels were measured by flow cytometry (D) Transfection of ASCT2 vector lowered the induction of ROS production induced by resveratrol. (E) Picture of $\gamma \mathrm{H} 2 \mathrm{AX}$ immunofluorescent staining of transfected C3A and SMCC7721 cells. (F) Quantification of $\gamma \mathrm{H} 2 \mathrm{AX}$ immunofluorescent staining by IOD/Area of transfected $\mathrm{C} 3 \mathrm{~A}$ and SMCC7721 cells. (G) Western blot assays were performed to determine the expression levels of mitochondria and kytoplasm cytochrome $\mathrm{C}$, caspase-9 and cleaved caspase-3 in transfected C3A and SMCC7721 cells. $\beta$-Actin was used as a loading control. ${ }^{*} \mathrm{P}<$ $0.05, * * P<0.01, * * * P<0.0001$ 
still need to be elucidated. In this report, we provide evidence demonstrating that resveratrol decreases the absorption of glutamine by reducing the expression of glutamine transporter ASCT2. Glutamine can be directly converted to glutathione, which is the primary reactive oxygen species (ROS) scavenging system in cells. Our subsequent studies demonstrate that resveratrol-induced chemosensitivity of cisplatin is associated with increase of ROS production, DNA damage and apoptosis induction in human hepatoma cells, suggesting resveratrol may sensitize human hepatoma cells to cisplatin chemotherapy via glutamine metabolism inhibition.

Extensive studies have shown that resveratrol has protective effects on nephrotoxicity, ototoxicity and heart damage induced by cisplatin (20-22). Resveratrol promotes cisplatin-induced apoptosis of lung cancer cells by regulating apoptosis signaling pathway in lung cancer cells, thereby inhibiting the proliferation of lung cancer cells (23). Our study shows that resveratrol enhances cisplatin toxicity in human hepatoma cells via an apoptosis-dependent mechanism. Resveratrol did not increase the killing effect of cisplatin on normal hepatic cells.

Glutaminolysis is presumably 10 -fold higher than that of any other amino acid in cancer cells. Many nutrient transporters have recently been found to play a new role in cancer chemosensitivity (24). Glutamine is transported into cancer cell mainly by solute carrier family 1 neutral amino acid transporter member 5 (SLC1A5, also known as ASCT2) (25). Membrane-anchored amino acid transporter SLC1A5 (ASCT2) mediates glutamine uptake in cancer cells. ASCT2 is expressed in liver cancer biopsies but is not detectable in normal hepatocytes. Six hepatoma cell lines (SK-Hep, HepG2, PLC/PRF/5, Hep3B, Focus and SMCC7721) were proven to be ASCT2-dependent in taking up glutamine several folds faster than normal hepatocytes (26). Previous studies have shown that ASCT2 is important for cell growth in several kinds of cancer cells (27-31). We found that the process where resveratrol enhancing cisplatin toxicity on human hepatoma cell lines involved the inhibition of glutamine metabolism in human hepatoma cell lines. In glutamine metabolic pathways, it was found that resveratrol can inhibit the uptake of glutamine via downregulating glutamine transporter ASCT2, which results in the inhibition of glutathione production. This indicated that resveratrol has the ability of inhibiting the glutamine metabolism of human hepatoma cell lines.

Cisplatin induces the production of ROS, which could lead to DNA damage. Cisplatin's anti-tumoral properties are mainly on the induction of DNA cross-links with the purine bases on the DNA (32), forming cisplatin-DNA adducts by damaging DNA and inhibiting DNA synthesis as well as it triggers the tumor suppressor protein p53 activation that leads to apoptosis. DNA damage results in phosphorylation and stabilization of p53 (33) that can transactivate genes involved in cell progression and apoptosis (34) and promote cisplatin- induced apoptosis by directly binding and counteracting the antiapoptotic function of Bcl-xL (35). DNA damage can initiate the intrinsic pathway, resulting in release of cytochrome-c from the mitochondria regulated by $\mathrm{Bcl}-2$ family activating procaspase-9 through the interaction with apoptosis promoting activating factor-1 (APAF-1) and formation of an active apoptosome complex. It has been found that resveratrol could enhance 5-fu anti-tumor activity by improving ROS production in colon cancer cells (36). Flow cytometric analyses demonstrate that resveratrol and cisplatin combined treatment leads to a significant increase in ROS production compared to resveratrol or cisplatin treated hepatoma cells. To quantify $\gamma \mathrm{H} 2 \mathrm{AX}$ - associated DNA damage, our $\gamma \mathrm{H} 2 \mathrm{AX}$ foci assay demonstrate that both resveratrol and cisplatin treatment result in a significant increase in the formation of $\gamma \mathrm{H} 2 \mathrm{AX}$ foci in hepatoma cells, and the resveratrol and cisplatin combined treatment results in much more substantial $\gamma \mathrm{H} 2 \mathrm{AX}$ foci formation than either resveratrol or cisplatin treatment alone. Western blot analyses showed that RV treatment has significant effect on the expression of mitochondria and kytoplasm cytochrome c, caspase-9 and activated caspase-3. These results support that the effect of resveratrol enhancing cisplatin toxicity may be related with increasing ROS-induced DNA damage.

In order to confirm that the synergistic effect of resveratrol with ciaplatin depends on the downregulation of ASCT2, we constructed ASCT2 recombinant expression plasmid, so that ASCT2 was highly expressed in C3A and SMCC7721 cells. Then we found that in both ASCT2 highly expressed cells, the effect of resveratrol enhancing cisplatin anti-tumor was significantly inhibited. All of these results demonstrated that the mechanism of resveratrol enhancing the antitumor effect of cisplatin was targeting at ASCT2. However, lack of in vivo data is the limit of this study. Further animal studies are required to examine whether combination therapy of CDDP and RV is beneficial for the treatment of HCC.

In conclusion, this study proves that the synergistic effect of resveratrol and cisplatin was first initiating the downregulation of ASCT2 by resveratrol, then inhibiting glutamine metabolism of human hepatoma cell lines to enhance cisplatin toxicity. In vivo studies are warranted to examine whether combination therapy of CDDP and RV could be applied in the clinics.

\section{MATERIALS AND METHODS}

\section{Cell culture}

Human hepatoma cell line C3A and SMCC7721 were purchased from American Type Culture Collection (ATCC). Human liver cell line LO2 was purchased from Shanghai Cell Library, CAS. All cells were cultured in DMEM with $10 \% \mathrm{FBS}$ and $100 \mathrm{u} / \mathrm{ml}$ of penicillin-streptomycin (Hyclone), at $37^{\circ} \mathrm{C}$ in an atmosphere of $5 \%$ $\mathrm{CO}_{2}$.

\section{Chemicals}

Resveratrol and cisplatin were purchased from Sigma-Aldrich Chemical Co. (St. Llouis, MO). Both drugs were dissolved in DMSO at a final concentration lower than $0.1 \%$.

Drug treatment and drug interaction effect test

C3A, SMCC7721 and LO2 cells were exposed to a range of drug 
concentrations for $24 \mathrm{~h}$ : resveratrol $(12.5 \mathrm{ug} / \mathrm{ml}, 25 \mathrm{ug} / \mathrm{ml}, 50 \mathrm{ug} / \mathrm{ml}$, $100 \mathrm{ug} / \mathrm{ml}, 200 \mathrm{ug} / \mathrm{ml})$, cisplatin $(0.625 \mathrm{ug} / \mathrm{ml}, 1.25 \mathrm{ug} / \mathrm{ml}, 2.5$ $\mathrm{ug} / \mathrm{ml}, 5 \mathrm{ug} / \mathrm{ml}, 10 \mathrm{ug} / \mathrm{ml}$ ), $12.5 \mathrm{ug} / \mathrm{ml}$ resveratrol $+0.625 \mathrm{ug} / \mathrm{ml}$ cisplatin, and $25 \mathrm{ug} / \mathrm{ml}$ resveratrol $+1.25 \mathrm{ug} / \mathrm{ml}$ cisplatin. Interaction of two drugs was evaluated by $\mathrm{CDI}$ (coefficient of drug interaction). $\mathrm{CDI}$ was calculated by the formula: $\mathrm{CDI}=\mathrm{AB} /(\mathrm{A}$ $\times B$ ), where $A B$ represents the cell viability after cells incubated with drug $A$ and $B$, while $A$ or $B$ represent cell viability after cells incubated with a single compound alone. $\mathrm{CDI}<1$ represents synergy of $A$ and $B, C D I=1$ represents additivity of $A$ and $B$, and CDI $>1$ represents antagonism of $A$ and $B$ (14).

\section{Cell viability assay}

Cell viability was measured by MTS assay. The cells were incubated with 10 ul of MTS agents (Promega; USA) in $100 \mathrm{ul}$ of medium for $4 \mathrm{~h}$. After incubation, the absorbance of each sample was measured at $490 \mathrm{~nm}$ with a multiscan MK3 spectrophotometer (Thermo Fisher Scientific; USA). The percentage of cell viability was calculated as the ratio of OD value of treated cells to that of control cells.

\section{Apoptosis assay}

Percentages of apoptotic cells were evaluated by Annexin V-FITC apoptosis Kit (Keygen, China) according to manufacturer's protocol. Flow cytometry was performed on a BD Calibur cytometer. Data were then obtained by Flowjo software.

\section{Western blot analysis}

Protein were extracted using cell lysis buffers and proteinase inhibitor. Protein concentrations were quantified using BCA method. Fifty ng of protein samples were diluted with $5 \times$ SDS and denature in boiling water for $5 \mathrm{~min}$. After electrophoresis, protein samples were transferred onto nitrocellulose filter membrane (NC membrane). The membrane was incubated in TBST solution that contains primary antibodies for $10 \mathrm{~min}$ at room temperature. Incubation with secondary antibody was prepared in the same way. Protein bands were detected using gel image processing system.

\section{Glutamine uptake assay}

Cells were washed twice with PBS. After adding phenol red-free DMEM to the plates to culture for $24 \mathrm{~h}$, the cells were collected for testing. Following the principle of glutamine conversion into glutamic acid and ammonia, the amount of glutamine can be calculated by measuring the amount of ammonia. According to the instructions of the glutamine measurement kit (Nanjing Jiancheng, China), the glutamine uptake by cells equals to the primary concentration of glutamine in phenol red free DMEM minus the concentration of glutamine of every group.

\section{Total glutathione assay}

According to the instructions of the glutathione measurement kit (Nanjing Jiancheng, China), total glutathione of cells treated with drugs at the concentrations indicated were assayed.

\section{Flow cytometric analysis of ROS}

ROS were measured by flow cytometric analysis. Cells were loaded with $50 \mathrm{uM}$ of dihydropyridine and incubated at $37^{\circ} \mathrm{C}$ for $30 \mathrm{~min}$. The excitation wavelength was $485 \mathrm{~nm}$ and the emission wavelength was $565 \mathrm{~nm}$.

\section{Immunofluorescent microscopy of $\gamma \mathrm{H} 2 \mathrm{AX}$ foci}

Cells were fixed with paraformaldehyde for $30 \mathrm{~min}$ and washed 3 times with PBS. Then the cells were permeabilized with $0.2 \%$ Triton X-100/PBS for 5 min. Slides were blocked with $10 \%$ normal goat serum for $30 \mathrm{~min}$ before incubation with mouse anti-phospho $\mathrm{H} 2 \mathrm{AX}$ monoclonal antibody for $2 \mathrm{~h}$ at room temperature or overnight at $4^{\circ} \mathrm{C}$ and thenincubated with Alexa Fluor 555-conjugated anti-mouse IgG secondary antibody (Invitrogen, USA) for $1 \mathrm{~h}$ at room temperature. Nuclei were counterstained with DAPI. Slides were then washed with PBS. Slides were briefly rinsed in water and air-dried prior to mounting.

\section{Over-expression of ASCT2}

Cells were transfected with empty pcDNA3.1 with Lipofectamine 2000 according to the manufacturer's instructions. Cells were incubated for $24 \mathrm{~h}$ to ensure adequate expression of ASCT2. The pcDNA3.1-ASCT2 was transfected into C3A and SMCC7721 cells.

\section{Statistical analysis}

Quantitative data were presented as mean \pm SD. Differences between groups were determined by Student's t-test. $\mathrm{P}<0.05$ was considered statistically significant.

\section{ACKNOWLEDGEMENTS}

This work was supported by the National High Technology Research and Development Program of China ("863" Program 2012AA020505), National Natural Science Foundation of China (8147875), Natural Science Foundation of Guangdong Province (2014A030312013); Science and Technology Planning Project of Guangdong Province (2014B020227002, 2015B090903069, 2015B020229002). Natural Science Foundation of Guangdong Province (2018A030313128, 2018A03031321,); National Key R\&D Program of China (2018YFA0108200).

\section{CONFLICTS OF INTEREST}

The authors have no conflicting interests.

\section{REFERENCES}

1. Llovet JM (2004) Treatment of hepatocellular carcinoma. Curr Treat Options Gastroenterol 7, 431-441

2. Llovet JM, Real MI, Montana X et al (2002) Arterial embolisation or chemoembolisation versus symptomatic treatment in patients with unresectable hepatocellular carcinoma: a randomized controlled trial. Lancet 359, 1734-1739

3. Lo CM, Ngan H, Tso WK et al (2002) Randomized controlled 
trial of transarterial lipiodol chemoembolization for unresectable hepatocellular carcinoma. Hepatology 35, 1164-1171

4. Murata S, Mine T, Sugihara F et al (2014) Interventional treatment for unresectable hepatocellular carcinoma. World J Gastroenterol 20, 13453-13465

5. Shaaban S, Negm A, Ibrahim EE et al (2014) Chemotherapeutic agent for the treatment of hepatocellular carcinoma: efficacy and mode of action. Oncol Rev 8, 25-35

6. Gupta SC, Kannappan R, Reuter S, Kim JH and Aqqarwal BB (2011) Chemosensitization of tumors by resveratrol. Ann NY Acad Sci 1215, 150-160

7. Sale S, Tunstall RG, Ruparelia KC, Potter GA, Steward WP and Gescher AJ (2005) Comparison of the effects of the chemopreventive agent resveratrol and its synthetic analog trans 3,4,5,4'-tetramethoxystilbene (DMU-212) on adenoma development in the Apc (Min+) mouse and cyclooxygenase- 2 in human-derived colon cancer cells. Int J Cancer 115, 194-201

8. Newsholme E, Crabtree B and Ardawi M (1985) The role of high rates of glycolysis and glutamine utilization in rapidly dividing cells. Biosci Rep 5, 393-400

9. Wellen KE, Lu C, Mancuso A et al (2010) The hexosamine biosynthetic pathway couples growth factor-induced glutamine uptake to glucose metabolism. Genes Dev 24, 27842799

10. Kueck A, Opipari AW, Griffith KA et al (2007) Resveratrol inhibits glucose metabolism in human ovarian cancer cells. Gynecol Oncol 107, 450-457

11. Park JB (2001) Inhibition of glucose and dehydroascorbic acid uptakes by resveratrol in human transformed myelocytic cells. J Nat Prod 64, 381-384

12. Hwang SO and Lee GM (2008) Nutrient deprivation induces autophagy as well as apoptosis in chinese hamster ovary cell culture. Biotechnol Bioeng 99, 678-685

13. Hassan $M$, Watari $H$, Abualmaaty A, Ohba $Y$ and Sakuragi $N$ (2014) Apoptosis and molecular targeting therapy in cancer. Biomed Res Int 2, 1-23

14. Chou TC and Talalay P (1984) Quantitative analysis of dose-effect relationships: the combined effects of multiple drugs or enzyme inhibitors. Adv Enzyme Regul 22, 27-55

15. Björklund M, Roos J, Gogvadze V and Shoshan M (2011) Resveratrol induces SIRT1- and energy-stress-independent inhibition of tumor cell regrowth after low-dose platinum treatment. Cancer Chemother Pharmacol 68, 1459-1467

16. Desoize B and Madoulet C (2002) Particular aspects of platinum compounds used at present in cancer treatment. Crit Rev Oncol Hematol 42, 317-325

17. Todd RC and Lippard SJ (2009) Inhibition of transcription by platinum antitumor compounds. Metallomics 1, 280-291

18. Wu YJ, Muldoon LL and Neuwelt EA (2005) The chemoprotective agent $\mathrm{N}$-acetylcysteine blocks cisplatininduced apoptosis through caspase signaling pathway. J Pharmacol Exp Ther 312, 424-431

19. Ma L, Li W, Wang R et al (2015) Resveratrol enhanced anticancer effects of cisplatin on non-small cell lung cancer cell lines by inducing mitochondrial dysfunction and cell apoptosis. Int J Oncol 47, 1460-1468

20. Wang J, He D, Zhang Q, Han Y, Jin S and Qi F (2009) Resveratrol protects against cisplatin-induced cardiotoxicity by alleviating oxidative damage. Cancer Biother Radiopharm $24,675-680$

21. Şimşek G, Tokgoz S, Vuralkan E, Caliskan M, Besalti O and Akin I (2013) Protective effects of resveratrol on cisplatin-dependent inner-ear damage in rats. Eur Arch Otorhinolaryngol 270, 1789-1793

22. Kim D, Jung Y, Lee J et al (2011) SIRT1 activation by resveratrol ameliorates cisplatin-induced renal injury through deacetylation of p53. Am J Physiol Renal Physiol 301, F427-F435

23. De Oliveira MR, Nabavi SF, Manayi A, Daglia M, Hajheydari Z and Nabavi SM (2016) Resveratrol and the mitochondria: from triggering the intrinsic apoptotic pathway to inducing mitochondrial biogenesis, a mechanistic view. Biochim Biophys Acta 1860, 727-745

24. Huang Y, Anderle P, Bussey KJ et al (2004) Membrane transporters and channels: role of the transportome in cancer chemosensitivity and chemoresistance. Cancer Res 64, 4294-4301

25. Wise DR and Thompson CB (2010) Glutamine addiction: a new therapeutic target in cancer. Trends BiochemSci 35, 427-433

26. Bode BP, Fuchs BC, Hurley BP et al (2002) Molecular and functional analysis of glutamine uptake in human hepatoma and liver-derived cells. Am J Physiol Gastrointest Liver Physiol 283, G1062-1073

27. Wang Q, Beaumont KA, Otte NJ et al (2014) Targeting glutamine transport to suppress melanoma cell growth. Int J Cancer 135, 1060-1071

28. Willems L, Jacque N, Jacquel A et al (2013) Inhibiting glutamine uptake represents an attractive new strategy for treating acute myeloid leukemia. Blood 122, 3521-3532

29. Hassanein M, Hoeksema MD, Shiota M et al (2013) SLC1A5 mediates glutamine transport required for lung cancer cell growth and survival. Clin Cancer Res 19, 560-570

30. Ren P, Yue M, Xiao D et al (2015) ATF4 and N-Myc coordinate glutamine metabolism in MYCN-amplified neuroblastoma cells through ASCT2 activation. J Pathol 235, 90-100

31. Kaira K, Sunose Y, Arakawa K et al (2015) Clinicopathological significance of ASC-amino acid transporter 2 (ASCT2) expression in pancreatic ductal carcinoma. Histopathology 66, 234-243

32. Brozovic A and Osmak M (2007) Activation of mitogenactivated protein kinases by cisplatin and their role in cisplatin-resistance. Cancer Lett 251, 1-16

33. Efeyan A and Serrano M (2007) p53: guardian of the genome and policeman of the oncogenes. Cell Cycle 6, 1006-1010

34. De Laurenzi $V$ and Melino G (2000) Evolution of functions within the p53/p63/p73 family. Ann NY Acad Sci 926, 90-100

35. Kutuk O, Arisan ED, Tezil T, Shoshan MC and Basaga H (2009) Cisplatin overcomes Bcl-2-mediated resistance to apoptosis via preferential engagement of Bak: critical role of Noxa-mediated lipid peroxidation. Carcinogenesis 30, 1517-1527

36. Santandreu FM, Valle A, Oliver J and Roca P (2011) Resveratrol potentiates the cytotoxic oxidative stress induced by chemotherapy in human colon cancer cells. Cell Physiol Biochem 28, 219-228 